\title{
Severe Hypertriglyceridemia in Pregnancy - A Case Report
}

\author{
ROKSHANAIVY ${ }^{1}$, RAWSHAN HOSNE JAHAN ${ }^{2}$, ANJUMAN ARA ${ }^{3}$
}

\begin{abstract}
:
We report a case of a 33 years primigravida who had regular antenatal check up and her pregnancy was uneventful till 30 weeks of pregnancy when she developed hypertension and her blood pressure was $150 / 90 \mathrm{~mm}$ of $\mathrm{Hg}$. At that time urinary protein was trace and she was treated conservatively. At 33 weeks of pregnancy, her blood pressure raised to 160/105 mm of $\mathrm{Hg}$ along with mild proteinuria. Serum creatinine and uric acid levels were within normal range. Ultrasonography revealed 32 weeks pregnancy with fetal weight $1800 \mathrm{gm}$ and $\mathrm{AFI} 7 \mathrm{~cm}$. The patient was treated conservatively. At 36 weeks of pregnancy, the patient perceived less fetal movement and Biophysical profile was found 6. Emergency caesarean section was done. During operation, blood was found pinkish in colour and after keeping the placenta on kidney dish, milky fluid was accumulated around the placenta. Urgently lipid profile of the patient was done and the report revealed high Triglyceride (3012 mg/dl) and cholesterol levels (348 mg/dl). The postoperative period was uneventful and she was treated with injection Heparin in the immediate postoperative period followed by low fat diet and lipid lowering agents.
\end{abstract}

Key words: pregnancy, hypertriglyceridemia.

\section{Introduction:}

During normal pregnancy, women show an increase in lipid levels of Triglyceride (TG) and total Cholesterol (TC) as gestational age progresses. Both TG and TC are taken up by the placenta and then metabolized and transported to the fetus in various forms. Both lipids are essential for the development of the fetus. ${ }^{1}$ The substantial modification of the lipid and lipoprotein metabolism occurs from second trimester of pregnancy. ${ }^{2}$ This is highly regulated response to satisfy energy and metabolic demands of the developing fetus. Pregnancy disorders, such as pre-eclampsia, are associated with a dysregulation of lipid metabolism manifesting in increased maternal plasma lipid levels. ${ }^{3}$ Pregnancy related hypertriglyceridemia is rare but it can be life threatening for some patients. Complications can include acute pancreatitis, hyperviscosity syndrome, pre-eclampsia and eclampsia. ${ }^{4}$ Acute pancreatitis may occur either during pregnancy, in $3^{\text {rd }}$ trimester or in the puerperium. Mortality is high for both mother, $21 \%$ and for the fetus, $20 \%{ }^{5}$

\section{Case report:}

A 33 years old primigravida was a booked patient and had regular antenatal check up in a private clinic. All of her physical findings and routine investigations were normal. Pregnancy was uneventful up to 30 weeks of pregnancy. Then she developed raised blood pressure (BP). Her BP was 150/90 mm of $\mathrm{Hg}$ and urinary protein was trace and initially was treated conservatively. At follow up visit at 33 weeks of pregnancy, her BP was found 160/ $105 \mathrm{~mm}$ of $\mathrm{Hg}$ along with mild proteinuria. Serum creatinine and uric acid levels were within normal range. Ultrasonography revealed 32 weeks pregnancy with fetal weight 1800 $\mathrm{gm}$ and AFI $7 \mathrm{~cm}$. The patient was treated conservatively and inj oradexon was given for lung maturity. At 36 weeks of pregnancy, the patient complained of less fetal movement and her biophysical profile was found 6. Emergency Caesarean section was done on $4^{\text {th }}$ August, 2010. During operation, blood was found pinkish in colour

1. Associate Professor (OB/GY) Shaheed Suhrawardy Medical College, Dhaka.

2. Senior Consultant (OB/GY) MCHTI, Azimpur, Dhaka

3. Registrar (OB/GY), Ibn Sina Medical College, Dhaka 


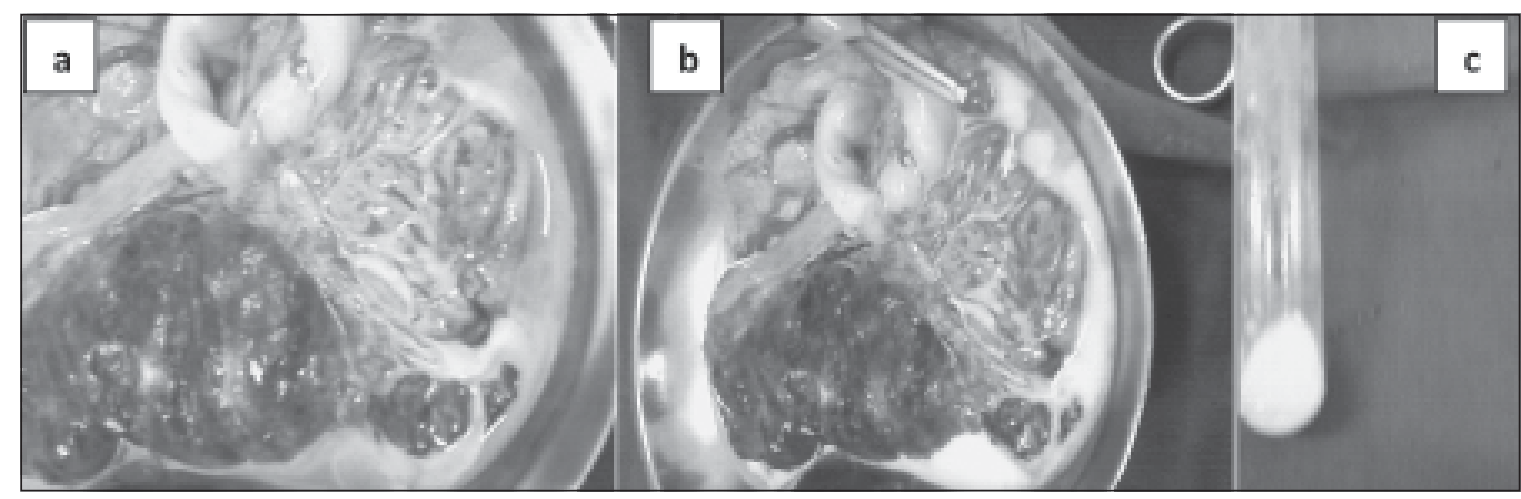

Fig.-1: a. Kidney dish with placenta \& pinkish blood around it, b. Kidney dish with placenta \& milky fluid around it, c. Test tube showing milky fluid.

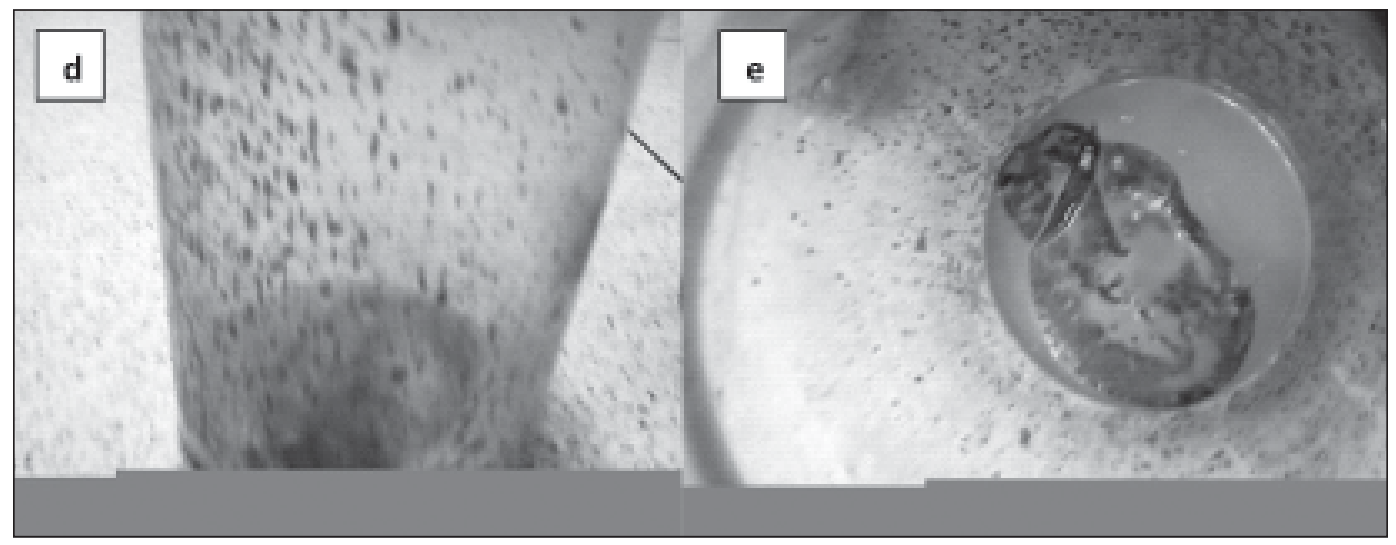

Fig.-2: $d \&$ e. Sucker bottle showing pinkish blood with small clot

and after keeping the placenta on the kidney dish, milky fluid was accumulated around the placenta.

Urgently lipid profile of the patient was done and triglyceride level was found very high, $3012 \mathrm{mg} / \mathrm{dl}$ and cholesterol level was $348 \mathrm{mg} / \mathrm{dl}$. Consultation regarding her hyperlipidaemic state was done with a physician and post-operatively the patient was treated with IV fluid and inj. Heparin, 5000 units sub cutaneously 8 hourly for 2 days. Baby's weight was $2 \mathrm{~kg}$ and kept in NICU for 2 days. Later on according to the physician's advice, she was treated with low dietary fat and lipid lowering drugs. Postoperative period was uneventful and was discharged on $6^{\text {th }}$ postoperative day. Her blood pressure became normal three weeks following delivery. She continued regular consultation with physician and since June, 2014 not taking any lipid lowering drug. Her last fasting lipid profile revealed cholesterol $170 \mathrm{gm} / \mathrm{dl}$ and triglyceride110 gm/dl.

\section{Discussion:}

During normal pregnancy, it is usual for blood lipids to increase significantly. Total cholesterol, HDL- and LDL cholesterol increase up-to $25-50 \%$, while triglyceride increases 2 to 4 times. $^{6}$ Diagnosis of hypertriglyceridemia for our patient was done after delivery. It was a retrospective diagnosis. Complications like pre-eclampsia with fetal growth restriction was present in our patient, which was probably due to hypertriglyceridemia. Vandenbrucke $\mathrm{L}$ et $\mathrm{al},{ }^{7}$ in their study described a case, where a woman at 37 weeks gestation presented with acute abdomen and fetal distress, and caesarean section was done. During caesarean section an abundant milky peritoneal fluid was noted. Later on she was diagnosed as having hypertriglyceridemia induced acute pancreatitis. Perrone $\mathrm{G}$ et al, ${ }^{5}$ reported a case of 36 weeks gestation with severe hypertriglyceridemia $(T G=3315 \mathrm{mg} / \mathrm{dl})$ and hypercholesterolemia (TC=425 mg/dl) and delivered 
by caesarean section. The baby's weight was $2670 \mathrm{gm}$ and APGAR score was 9 in $1^{\text {st }}$ minute. The patient was post-operatively treated with IV fluid and plasma exchange. In our patient, no significant change was noticed in peritoneal fluid but milky fluid was accumulated around the placenta after keeping in kidney dish. The lipid profile of our patient was almost same as that of Perrone $G$ et al. Athyros VG et al, ${ }^{8}$ stated that extreme elevation of TG, usually above $1000 \mathrm{mg} / \mathrm{dl}$, may cause acute pancreatitis and all the sequelae of that condition. However, many patients tolerate TG of $4000 \mathrm{mg} / \mathrm{dl}$ or higher without developing symptoms. It is very important to remember that Pregnancy disorders, such as preeclamsia, are associated with a dysregulation of lipid metabolism manifesting in increased maternal plasma lipid levels. ${ }^{3}$

\section{Conclusion:}

Pregnancy related hypertriglyceridemia is rare but complications like pre-eclampsia, hyperviscosity syndrome, atherosclerosis and life threatening acute pancreatitis can occur. Fetal morbidity and mortality are also high. So, early diagnosis and proper management can minimize the adverse outcomes of both mother and baby.

\section{Recommendation:}

The fasting lipid profile is to be done in Patients with pre-eclampsia, otherwise diagnosis of hypertriglyceridemia induced pre-eclampsia may be missed and therefore, there may be more serious consequences of hypertriglyceridemia on pregnancy.

\section{References:}

1. Tanja GM, NathalieK,Barbara A, et al. Maternal Lipid profile during early pregnancy and pregnancy complications and outcomes: The ABCD Study. The Journal of Clinical Endocrinology and Metabolism. 2012; 97(11):0021-972X. Check whether it is correct

2. Lippi G, Albiero A, Montagnana M, et al. Lipid and lipoprotein profile in physiological pregnancy. Clin. Lab, 2007; 53: XXX-XXX Check whether it is correct

3. Huang $X$, jain $A$, Baumann $M$, et al. Increased placental phospholipid levels in pre-eclamptic pregnancies. Int J Mol Sci.2013 Feb 6;14(2): 3487- 99 What stands for 6 ?

4. Goldberg AS and Hegele RA. Severe hypertriglyceridemia in pregnancy. Journal of Clinical Endocrinol Metab. 2012; 97(8):2589- 96.

5. Perrone $G$ and Critelle C. Severe hypertriglyceridemia in pregnancy. A clinical case report. Minerva Gynecol. 1996; 40(12):573- 6

6. Dukic A, ZivancevicS, et al. Hyperlipidemia and pregnancy. Med Preql. 2009; 62(3):80- 84

7. Vandenbroucke L, Seconda S, et al. Acute parcreatitis induced by major hypertriglyceridemiaduring pregnancy. A case report. J Gynecol Obstet Biol Record. 2009; 38(5):436- 439

8. Athyros VG, Giouslesme OI, et al. Long term follow up of patient with acute hypertriglyceridemia induced pancreatitis. J ClinGastroenterol. 2002; 34(4): 472-5. 